\title{
The Relationship of Anti-Mullerian Hormone in Polycystic Ovary Syndrome Patients with Different Subgroups
}

This article was published in the following Dove Press journal:

Diabetes, Metabolic Syndrome and Obesity: Targets and Therapy

\section{Yu Ran \\ Qiang Yi \\ Cong $\mathrm{Li}$ (iD}

Department of Gynecology, The First Affiliated Hospital of Chongqing Medical University, Chongqing, People's Republic of China
Correspondence: Cong $\mathrm{Li}$

Department of Gynecology, The First Affiliated Hospital of Chongqing Medical University, No. I YouYi Road, YuZhong District, Chongqing, People's Republic of China

Tel +8615334506105

$\mathrm{Fax}+8602389011080$

Email b05104@126.com
Purpose: To explore the value of anti-Mullerian hormone (AMH) in patients with polycystic ovary syndrome (PCOS) with different phenotypes and ages, and to identify the relationship between hyperandrogenism (HA) and polycystic ovary morphology (PCOM), in a Chinese cohort.

Methods: A total of 2262 women ( 1631 with PCOS and 631 controls) were enrolled. The serum $\mathrm{AMH}$ and total testosterone (TT) were analyzed, the AMH levels of each subgroup were compared, and the value of each phenotype and age group of patients with PCOS was evaluated. Results: The level of AMH in women with $\mathrm{PCOS}$ (mean $\pm \mathrm{SD}, 8.63 \pm 4.73 \mathrm{ng} / \mathrm{mL}$ ) was higher than that in controls $(5.57 \pm 3.31 \mathrm{ng} / \mathrm{mL})(P<0.01)$. The level of AMH in the PCOM subgroup $(11.19 \pm 6.4$ $\mathrm{ng} / \mathrm{mL})$ was significantly higher than that in the HA subgroup $(8.58 \pm 4.74 \mathrm{ng} / \mathrm{mL})(P<0.01)$, and both were higher than that in controls $(P<0.01)$. AMH was higher in PCOS patients than in controls, but the same values were found in subgroups of PCOS patients under 30 years old.

Conclusion: AMH changed in different subgroups of PCOS, which was the possible reason why $\mathrm{AMH}$ was not a diagnostic indicator. However, AMH could help to differentiate between clinical subgroups, as it was strongly related with PCOM but not with HA. AMH changed substantially with age, but was stable in PCOS patients under 30 years old.

Keywords: anti-Mullerian hormone, polycystic ovary syndrome, hyperandrogenism, polycystic ovary morphology

\section{Introduction}

Polycystic ovary syndrome (PCOS) is one of the most common endocrine disorders, affecting $8-13 \%$ of women of reproductive age. It is characterized by anovulation, hyperandrogenism (HA), and polycystic ovary morphology (PCOM). ${ }^{1}$ There are many phenotypes of the syndrome, including hyperandrogenism combined with ovulatory dysfunction, hyperandrogenism combined with polycystic ovarian morphology, ovulatory dysfunction combined with polycystic ovarian morphology, oligo-ovulation combined with polycystic ovarian morphology, and hyperandrogenism alone. Because of the large individual differences between women with PCOS, there are no exact effects of clinical treatment. Moreover, there are currently no relevant specific serological markers to identify subgroups for specific therapy initially. As a member of the transforming growth factor- $\beta$ family, anti-Mullerian hormone $(\mathrm{AMH})$, produced by granulosa cells in preantral and small antral ovarian follicles, can reflect the ovarian reserve and ovarian function. ${ }^{2}$ Serum AMH levels in PCOS are elevated two- to three-fold 
and are positively correlated with PCOM and serum androgen levels; ${ }^{3,4}$ therefore, AMH may be a potential marker for the diagnosis of PCOS. ${ }^{5}$ Although there are a number of studies and consensus on the relationship between AMH and PCOS, it is not yet used as a diagnostic criterion. Whether this is related to the presence of variability in the PCOS subgroup remains unclear. Therefore, this study was carried out to investigate the distribution of AMH between subgroups of young PCOS patients in a Chinese cohort, to better understand the possible diagnostic and follow-up value of AMH in PCOS.

\section{Patients and Methods Study Population}

The study included 2262 women with complaints of menstrual disorders, aged 16-29 years, in the outpatient gynecology department of The First Affiliated Hospital of Chongqing Medical University, from May 2018 to May 2020. The population was divided into a PCOS group of 1631 cases (1161 in the HA subgroup, 470 in the PCOM subgroup, and 399 in the HA+PCOM subgroup) and a control group of 631 cases, according to the Rotterdam criteria. ${ }^{6}$

The Rotterdam criteria for PCOS state that two out of the following three criteria must be present: 1) oligo- or anovulation (OA) is a necessary condition for diagnosis; plus another one of the following two criteria: 2) clinical or biochemical hyperandrogenemia; or 3) PCOM (presence of 12 follicles measuring 2-9 $\mathrm{mm}$ in diameter in each ovary and/or increased ovarian volume $[>10 \mathrm{~mL}]$ ) under ultrasound.

The patient groups comprised the PCOS subphenotypes of $\mathrm{OA}+\mathrm{HA}$ (irrespective of PCOM status), OA $+\mathrm{PCOM}$ (irrespective of $\mathrm{HA}$ status), and $\mathrm{OA}+\mathrm{HA}$ +PCOM groups. The controls were patients who had menstrual disorders only but did not have PCOM on ultrasound or the clinical or biochemical manifestations of HA.

The exclusion criteria were: 1) endocrine diseases other than PCOS that cause ovulation disorders, such as hyperprolactinemia, congenital dysfunction of the adrenal cortex, thyroid disorders, Cushing syndrome and disease, and tumors of the pelvic organs; 2) pregnancy; 3) total testosterone (TT) $<0.1 \mathrm{ng} / \mathrm{mL}$ or $\mathrm{AMH} \geq 23.5 \mathrm{ng} / \mathrm{mL}$; 4) over 30 years old; 5) a history of using drugs such as insulin sensitizers and oral contraceptives within 3 months.

\section{Study Methods}

TT and AMH were measured on days 2-4 of the spontaneous menstrual cycle or on any day for women with amenorrhea. Serum AMH and TT levels were measured by a electrochemiluminescence method using the Beckman DXI800 instrument. HA was defined as TT $\geq 0.5 \mathrm{ng} / \mathrm{mL}$ in our study.

Vaginal ultrasound was performed in the early follicular period (for women with regular menstruation) or in the state of no dominant follicle (transabdominal ultrasound was performed if the woman was not sexually active), measuring the length, cross diameter, front and rear diameters of the ovaries, and the size and number of follicles in each ovary. PCOM was defined as the presence of 12 follicles measuring $2-9 \mathrm{~mm}$ in diameter in each ovary and/or increased ovarian volume $(>10 \mathrm{~mL})$.

The study was approved by the Ethics Committee of The First Affiliated Hospital of Chongqing Medical University. Because this was a retrospective observational study with no intervention, formal ethical approval and written consent were not required. The study protocol was conducted in accordance with the Declaration of Helsinki.

\section{Statistical Analysis}

Analyses were performed with using SPSS 26.0 statistics software. The Shapiro-Wilk test was used to test whether the variables fitted a normal distribution. Comparison of variables with a normal distribution was performed by the parametric Student's $t$-test, while continuous variables were presented as the mean $\pm \mathrm{SD}$. Parameters that did not fit the normal distribution (according to the Shapiro-Wilk test) were analyzed using the median and interquartile range and the Mann-Whitney $U$-test. $P$ values of $<0.05$ were considered statistically significant.

\section{Results}

\section{Comparison of AMH Levels Between PCOS (and Subgroups) and Control Group}

The level of AMH in PCOS $(8.63 \pm 4.73 \mathrm{ng} / \mathrm{mL})$ was higher than that in controls $(5.57 \pm 3.31 \mathrm{ng} / \mathrm{mL})(P<0.01)$. The AMH levels in the HA, PCOM, and HA+PCOM subgroups were $8.58 \pm 4.74,11.19 \pm 6.4$, and $11.45 \pm 4.97$ $\mathrm{ng} / \mathrm{mL}$, respectively, and were higher than that in controls $(P<0.01)$ (Table 1). 
Table I AMH Levels of the Different Groups

\begin{tabular}{|l|c|c|c|c|}
\hline Group & $\mathbf{n}$ & AMH $(\mathbf{n g} / \mathbf{m L})$ & $\boldsymbol{Z}$ & $\boldsymbol{P}$ \\
\hline Controls & $63 \mathrm{I}$ & $5.57 \pm 3.31$ & & \\
PCOS & $163 \mathrm{I}$ & $8.63 \pm 4.73$ & 14.652 & $<0.0 \mathrm{I}$ \\
HA & 1560 & $8.58 \pm 4.74$ & 14.264 & $<0.0 \mathrm{I}$ \\
PCOM & 470 & $11.19 \pm 6.4$ & 18.844 & $<0.0 \mathrm{I}$ \\
PCOM+HA & 399 & $11.45 \pm 4.97$ & 18.382 & $<0.0 \mathrm{I}$ \\
\hline
\end{tabular}

Notes: The table shows the comparison between the PCOS group (and subgroups) and the control group. The AMH levels in the PCOS group, and in the HA, PCOM, and $\mathrm{HA}+\mathrm{PCOM}$ subgroups, were all higher than that in controls $(P<0.0 \mathrm{I})$.

Abbreviations: $\mathrm{AMH}$, anti-Mullerian hormone; PCOS, polycystic ovary syndrome; HA, hyperandrogenism; PCOM, polycystic ovary morphology.

\section{Comparison of AMH Levels Between PCOS Subgroups}

The level of AMH in the PCOM subgroup (11.19 $\pm 6.4 \mathrm{ng} /$ $\mathrm{mL})$ was significantly higher than that in the HA subgroup $(8.58 \pm 4.74 \mathrm{ng} / \mathrm{mL})(P<0.01)$. The level of $\mathrm{AMH}$ in the PCOM+HA subgroup $(11.45 \pm 4.97 \mathrm{ng} / \mathrm{mL})$ was significantly higher than that in the HA subgroup $(P<0.01)$. The level of AMH in the PCOM+HA subgroup $(11.45$ $\pm 4.97 \mathrm{ng} / \mathrm{mL}$ ) was higher than that in the PCOM subgroup, but this difference was not statistically significant $(P=0.459)$ (Table 2$)$.

\section{AMH Levels in Different Age Groups}

The AMH levels in the age groups 16-19, 20-25, and 26-29 years old in PCOS patients and controls are listed in Table 3. The changes in AMH level were roughly the same between PCOS patients and controls; they all increased at first and then decreased, reaching the highest level in 20-25-year-old $\mathrm{s}$, at $9.00 \pm 4.89$ and $6.14 \pm 3.31 \mathrm{ng} / \mathrm{mL}$, respectively.

\section{Discussion}

Differences in AMH levels between the different subgroups of PCOS may be a reason why AMH is not useful as a diagnostic indicator. However, $\mathrm{AMH}$ differed between

Table 2 AMH Levels of the Different PCOS Subgroups

\begin{tabular}{|l|c|c|c|c|}
\hline Group & $\mathbf{n}$ & AMH (ng/mL) & $\boldsymbol{Z}$ & $\boldsymbol{P}$ \\
\hline PCOM & 470 & $11.19 \pm 6.4$ & 0.741 & $0.459 *$ \\
HA & 1560 & $8.58 \pm 4.74$ & 10.443 & $<0.01^{\#}$ \\
PCOM+HA & 399 & $11.45 \pm 4.97$ & 10.359 & $<0.01^{\&}$ \\
\hline
\end{tabular}

Notes: *PCOM vs PCOM+HA; ${ }^{*} \mathrm{HA}$ vs PCOM+HA; ${ }^{2} \mathrm{PCOM}$ vs HA. The table shows the comparison between the three PCOS subgroups. The AMH level in the $\mathrm{HA}+\mathrm{PCOM}$ subgroup was higher than that in the HA group $(P<0.0 \mathrm{I})$ and that in the PCOM group $(P=0.447)$. The AMH level in the PCOM subgroup was higher than that in the $H A$ subgroup $(P<0.01)$.

Abbreviations: $\mathrm{AMH}$, anti-Mullerian hormone; PCOS, polycystic ovary syndrome; PCOM, polycystic ovary morphology; HA, hyperandrogenism.
Table 3 AMH Levels of Age-Stratified Groups of PCOS Patients and Controls

\begin{tabular}{|l|l|l|l|l|l|}
\hline \multirow{2}{*}{$\begin{array}{l}\text { Age } \\
\text { (years) }\end{array}$} & \multicolumn{2}{|l|}{$\mathbf{N}$} & \multicolumn{2}{l|}{ AMH $(\mathrm{ng} / \mathrm{mL})$} & \multirow{2}{*}{$\boldsymbol{P}$} \\
\cline { 2 - 5 } & PCOS & Controls & PCOS & Controls & \\
\hline $16-19$ & 226 & 103 & $8.84 \pm 4.52$ & $5.98 \pm 3.62$ & $<0.01$ \\
$20-25$ & 775 & 218 & $9.00 \pm 4.89$ & $6.14 \pm 3.31$ & $<0.01$ \\
$26-29$ & 630 & 310 & $8.10 \pm 4.56$ & $5.03 \pm 3.11$ & $<0.01$ \\
N & 1631 & 631 & $8.63 \pm 4.73$ & $5.57 \pm 3.31$ & \\
\hline
\end{tabular}

Notes: The table shows the AMH levels in different age groups of PCOS patients and controls. The change in AMH with age in PCOS is roughly the same as that in controls, reaching the highest level in the 20-25-year-old age group.

Abbreviations: AMH, anti-Mullerian hormone; PCOS, polycystic ovary syndrome.

the clinical subgroups, being strongly related to PCOM but not to HA. AMH levels changed greatly with age, but were stable in PCOS patients under 30 years old.

\section{AMH Levels and PCOS}

$\mathrm{AMH}$ is secreted by the granulosa cells in the preantral and small antral follicles. Several studies have shown that the level of AMH is two- to three-fold higher in women with PCOS than in healthy women of childbearing age, probably owing to the increased follicular mass or follicular hypersecretion. ${ }^{3,4}$ Studies have also suggested that the overexpression of AMHR2, an intrinsic dysregulation of the granulosa cells themselves, may be related to excessive AMH. ${ }^{7,8}$ Another study found that AMH receptors expressed in human gonadotropin-releasing hormone (GnRH) neurons can directly increase GnRH-dependent luteinizing hormone (LH) secretion, thus favoring AMH production. ${ }^{9}$ Serum $\mathrm{AMH}$ detection, as a non-invasive method, is not affected by the menstrual cycle or the non-fasting state, so it has potential as a new marker for the diagnosis of PCOS. Possible reasons for $\mathrm{AMH}$ not having been used as a diagnostic marker before nowinclude the different methods of detection, the influence of age, and the body mass index (BMI). ${ }^{10-12}$ In this clinical analysis, the variation in AMH in each subgroup may also be one of the reasons why it cannot be used as a diagnostic marker of PCOS, since AMH was significantly affected by different characteristics of PCOS. Without PCOM, the mean value of AMH was the same for PCOS patients and healthy controls; however, there are no clear cut-off values for AMH concentrations as for total and free testosterone, especially in adolescents. ${ }^{13}$ All of these factors contribute to the lack of standardization and appropriate cut-offs for the different conditions. ${ }^{14}$

Our study showed that women with PCOS had significantly higher AMH levels compared with controls, and 
this was true even for women who did not have HA or PCOM. Our study also demonstrated that AMH was positively correlated with the severity of PCOS phenotype, which was consistent with previous studies. ${ }^{10}$ The ovulatory dysfunction with HA phenotype (1560 cases) was the most prevalent form in our Chinese patient cohort, the ovulatory dysfunction with PCOM phenotype (470 cases) was less common, and the classic phenotype (399 cases) having all three features of the syndrome was seen the least but had the highest mean AMH level, as we expected. Furthermore, the diagnostic value of AMH was limited for the classic phenotype, possibly because the presence of irregular cycles and HA was sufficient for the diagnosis. ${ }^{15}$ Although researchers in a study involving 392 Turkish women with PCOS found that $\mathrm{AMH}$ had poor to fair ability to diagnose the syndrome in $\mathrm{OA}+\mathrm{HA}$ and $\mathrm{OA}$ + PCOM phenotypes, ${ }^{16}$ the result may not be applicable to our Chinese PCOS cohort. In our population, especially in the only HA (1161/1631) and PCOM (71/1631) subsets, the $\mathrm{AMH}$ level was obviously higher than that in controls. In those two subgroups, the $\mathrm{AMH}$ level could reflect independently the presence of HA and PCOM, so AMH still had a certain auxiliary diagnostic value for PCOS.

\section{Relationship Between $\mathrm{AMH}$ and $\mathrm{HA}$ or PCOM}

Our study indicated that the increase in $\mathrm{AMH}$ was the result of $\mathrm{HA}$ and $\mathrm{PCO}$ together, consistent with previous research. ${ }^{17}$ The serum AMH in the PCOM+non-HA group was higher than that in the HA+non-PCOM group (9.57 \pm 4.3 vs $7.6 \pm 4.23 \mathrm{ng} / \mathrm{mL}$ ), which showed that PCOM had a strong relationship with intrinsic secretion of $\mathrm{AMH}$. AMH could be used as a more sensitive alternative biomarker for follicular number per ovary (FNPO) in the diagnosis of PCOM, ${ }^{18}$ and, to some extent, could also remedy the false-negative results of ultrasound examination (including the effect of transabdominal ultrasound on follicular count in asexual women, and particularly in obese patients); conversely, with the advances in ultrasound equipment, the new international guidelines have redefined the PCOM cut-off to a threshold of $\geq 20$ FNPO, ${ }^{19}$ which could reduce the diagnostic rate of PCOS. Therefore, AMH seems likely to replace FNPO as the marker of PCOM because it can not only represent the increasing basic follicles but also reflect the pathological state of hypersecretion of the follicles to a certain extent.
HA had a weaker relationship in our results, which differed from some previous findings which reported a positive correlation between serum $\mathrm{AMH}$ and $\mathrm{TT}^{20,21}$ and found that AMH levels were higher in PCOS when

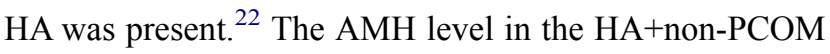
group (1161/1631) was higher than that in the healthy control group $(7.6 \pm 4.23$ vs $5.57 \pm 3.31 \mathrm{ng} / \mathrm{mL})$ in our study. Androgens stimulate follicle-stimulating hormone receptor (FSHR) expression and promote follicular growth, which leads to elevated AMH production. The elevated AMH suppresses aromatase expression in granulosa cells, and hinders the transformation of androgen to estrogen, which contributes to the increased androgen level. $^{23}$ This mutually reinforced interaction between $\mathrm{AMH}$ and HA may be the reason for PCOS.

\section{$\mathrm{AMH}$ and Age}

As a biomarker of the number of ovarian antrum follicles, AMH declines with increasing age. ${ }^{24}$ Since the AMH level is obviously reduced after 30 years of age, we chose patients under 30 years old, to more accurately detect the disparity in AMH between the PCOS and non-PCOS cohorts without interference from age factors. Despite the lack of an international standard for $\mathrm{AMH}$, according to the recent international guideline, the areas under the ROC curve of $\mathrm{AMH}$ for the diagnosis of PCOS range from 0.66 to 0.994 and the threshold cut-off values range from 10 to $57 \mathrm{pmol} / \mathrm{L}$; $^{1}$ thus, significant heterogeneity exists and it can be confusing for clinicians to refer to these guidelines. In our study, we divided AMH levels into three age groups, 16-19, 21-25, and 26-29 years old, which could provide more detailed standards for PCOS diagnosis. Moreover, another study found that AMH levels fall with increasing age in women of reproductive age with PCOS as well as in healthy controls. ${ }^{25}$ In our study, AMH levels increased at first in all age groups, reaching the highest level in the 20-25-year-old and then decreasing in 26-29-yearold women, and the same changes were seen in the control group. AMH levels were high in adolescence, and to some extent they may overlap with levels in PCOS patients, making it difficult to differentiate PCOS from controls using AMH levels. $^{26}$ Therefore, it would be helpful to have different standards for certain age groups to diagnose PCOS, although more detailed age segments and larger population samples are needed.

\section{Possible Reasons for AMH Variation}

An abnormal lipid profile and insulin resistance are common metabolic disorders in PCOS patients. $^{27}$ The 
relationship of AMH with these metabolic factors remains controversial with the current data. A previous study demonstrated that there was a significant negative correlation between AMH level and homeostasis model assessment of insulin resistance (HOMA-IR) or triglyceride levels, and a positive correlation between AMH and high-density lipoprotein cholesterol (HDL-C) or serum adiponectin level, so that AMH is also a potential cardiometabolic risk marker in women with $\mathrm{PCOS}^{28}$ Although the correlation between $\mathrm{AMH}$ and $\mathrm{BMI}$ remains unclear, the associations between $\mathrm{AMH}$ and metabolic syndrome were modified by BMI; hence, the women who had PCOS with normal BMI did not have an increased risk of metabolic syndrome. ${ }^{29}$ The present study is a retrospective study, and owing to the mobility of outpatients and incomplete data, metabolic markers were not included in the clinical data and the corresponding causal analysis data could not be obtained from this study; we will confirm these data in subsequent experiments.

\section{Conclusion}

In summary, our preliminary study confirmed the diagnostic value of $\mathrm{AMH}$ in PCOS, especially in subgroups of PCOS; the presence of PCOM has a closer relationship with the AMH level than HA, which means that the AMH level could also play a role in predicting clinical features and even in guiding the treatment of PCOS, besides in diagnosis. However, since the specificity and sensitivity are not high when using only the AMH level for the diagnosis of PCOS in young patients, according to a 2016 analysis, ${ }^{30}$ it is essential to establish different diagnostic thresholds according to age or BMI, and even ethnicity and detection methods, which could make the diagnostic value of AMH more costeffective. It is also necessary to combine these factors with glucose and lipid metabolism factors and to estimate the potential value of cardiometabolic risk markers in our further research.

\section{Abbreviations}

PCOS, polycystic ovary syndrome; HA, hyperandrogenism; PCOM, polycystic ovary morphology; AMH, anti-Mullerian hormone; OA, oligo/anovulation; TT, total testosterone; BMI, body mass index; FNPO, follicular number per ovary; FSHR, follicle-stimulating hormone receptor; HOMA-IR, homeostasis model assessment of insulin resistance; HDL-C, high-density lipoprotein cholesterol.

\section{Data Sharing Statement}

The datasets used and/or analyzed during the current study are available from the corresponding author on reasonable request.

\section{Ethics Approval and Consent to Participate}

The study was approved by the Ethics Committee of The First Affiliated Hospital of Chongqing Medical University (Scientific research ethics of 2020: number 2020-637). We confirm that the data was anonymized or maintained with confidentiality. Because this was a retrospectively observational study with no intervention, formal ethical approval and written consent were not required. The study protocol was conducted in accordance with the Declaration of Helsinki.

\section{Author Contributions}

All authors made a significant contribution to the work reported, whether that is in the conception, study design, execution, acquisition of data, analysis and interpretation, or in all these areas; took part in drafting, revising or critically reviewing the article; gave final approval of the version to be published; have agreed on the journal to which the article has been submitted; and agree to be accountable for all aspects of the work.

\section{Funding}

No funding was obtained for this study.

\section{Disclosure}

All authors declare no conflicts of interest.

\section{References}

1. Teede H, Misso M, Tassone EC, et al. Anti-Mullerian hormone in PCOS: a review informing international guidelines. Trends Endocrinol Metab. 2019;30(7):467-478. doi:10.1016/j.tem.2019.04.006

2. Broer SL, Broekmans FJ, Laven JS, et al. Anti-Mullerian hormone: ovarian reserve testing and its potential clinical implications. Hum Reprod Update. 2014;20(5):688-701. doi:10.1093/humupd/dmu020

3. Pigny P, Merlen E, Robert Y, et al. Elevated serum level of anti-mullerian hormone in patients with polycystic ovary syndrome: relationship to the ovarian follicle excess and to the follicular arrest. J Clin Endocrinol Metab. 2003;88(12):5957-5962. doi:10.1210/ jc. 2003-030727

4. Pellatt L, Hanna L, Brincat M, et al. Granulosa cell production of anti-Mullerian hormone is increased in polycystic ovaries. J Clin Endocrinol Metab. 2007;92(1):240-245. doi:10.1210/jc.2006-1582

5. Streuli I, Fraisse T, Pillet C, et al. Serum antimullerian hormone levels remain stable throughout the menstrual cycle and after oral or vaginal administration of synthetic sex steroids. Fertil Steril. 2008;90 (2):395-400. doi:10.1016/j.fertnstert.2007.06.023 
6. Geisthovel F. A comment on the European Society of Human Reproduction and Embryology American Society for Reproductive Medicine consensus of the polycystic ovarian syndrome. Reprod Biomed Online. 2003;7(6):602-605. doi:10.1016/S1472-6483(10) 62081-0

7. Alebic MS, Stojanovic N, Duhamel A, et al. The phenotypic diversity in per-follicle anti-Mullerian hormone production in polycystic ovary syndrome. Hum Reprod. 2015;30(8):1927-1933. doi:10.1093/humrep/dev131

8. Pierre A, Taieb J, Giton F, et al. Dysregulation of the anti-Mullerian hormone system by steroids in women with polycystic ovary syndrome. J Clin Endocrinol Metab. 2017;102(11):3970-3978. doi:10.1210/jc.2017-00308

9. Cimino I, Casoni F, Liu X, et al. Novel role for anti-Mullerian hormone in the regulation of $\mathrm{GnRH}$ neuron excitability and hormone secretion. Nat Commun. 2016;7:10055. doi:10.1038/ncomms10055

10. Lefebvre T, Dumont A, Pigny P, et al. Effect of obesity and its related metabolic factors on serum anti-Mullerian hormone concentrations in women with and without polycystic ovaries. Reprod Biomed Online. 2017;35(3):325-330. doi:10.1016/j.rbmo.2017.05.013

11. Simoes-Pereira J, Nunes J, Aguiar A, et al. Influence of body mass index in anti-Mullerian hormone levels in 951 non-polycystic ovarian syndrome women followed at a reproductive medicine unit. Endocrine. 2018;61(1):144-148. doi:10.1007/s12020-018-1555-y

12. Piouka A, Farmakiotis D, Katsikis I, et al. Anti-Mullerian hormone levels reflect severity of PCOS but are negatively influenced by obesity: relationship with increased luteinizing hormone levels. $\mathrm{Am}$ J Physiol Endocrinol Metab. 2009;296(2):E238-E243. doi:10.1152/ ajpendo.90684.2008

13. Khashchenko E, Uvarova E, Vysokikh M, et al. The relevant hormonal levels and diagnostic features of polycystic ovary syndrome in adolescents. J Clin Med. 2020;9(6):1831. doi:10.3390/jcm9061831

14. Escobar-Morreale HF. Polycystic ovary syndrome: definition, aetiology, diagnosis and treatment. Nat Rev Endocrinol. 2018;14 (5):270-284. doi:10.1038/nrendo.2018.24

15. Zijiang Chen QT. Guidelines for the diagnosis and treatment of polycystic ovary syndrome in China. Chin $J$ Obstet Gynecol. 2018;53(01):2-6.

16. Bozdag G, Mumusoglu S, Coskun ZY, et al. Anti-Mullerian hormone as a diagnostic tool for PCOS under different diagnostic criteria in an unselected population. Reprod Biomed Online. 2019;39(3):522-529. doi:10.1016/j.rbmo.2019.04.002

17. Karakas SE. New biomarkers for diagnosis and management of polycystic ovary syndrome. Clin Chim Acta. 2017;471:248-253. doi:10.1016/j.cca.2017.06.009

18. Dumont A, Robin G, Dewailly D. Anti-mullerian hormone in the pathophysiology and diagnosis of polycystic ovarian syndrome. Curr Opin Endocrinol Diabetes Obes. 2018;25(6):377-384. doi:10.1097/ MED.0000000000000445
19. Teede HJ, Misso ML, Costello MF, et al. Recommendations from the international evidence-based guideline for the assessment and management of polycystic ovary syndrome. Hum Reprod. 2018;33 (9):1602-1618. doi:10.1093/humrep/dey256

20. Lv PP, Jin M, Rao JP, et al. Role of anti-Mullerian hormone and testosterone in follicular growth: a cross-sectional study. $B M C$ Endocr Disord. 2020;20(1):101. doi:10.1186/s12902-020-00569-6

21. Sova H, Unkila-Kallio L, Tiitinen A, et al. Hormone profiling, including anti-Mullerian hormone $(\mathrm{AMH})$, for the diagnosis of polycystic ovary syndrome (PCOS) and characterization of PCOS phenotypes. Gynecol Endocrinol. 2019;35(7):595-600. doi:10.1080/ 09513590.2018.1559807

22. Wiweko B, Maidarti M, Priangga MD, et al. Anti-mullerian hormone as a diagnostic and prognostic tool for PCOS patients. J Assist Reprod Genet. 2014;31(10):1311-1316. doi:10.1007/s10815-0140300-6

23. Jacob SL, Field HP, Calder N, et al. Anti-Mullerian hormone reflects the severity of polycystic ovary syndrome. Clin Endocrinol (Oxf). 2017;86(3):395-400. doi:10.1111/cen.13269

24. Dewailly D, Andersen CY, Balen A, et al. The physiology and clinical utility of anti-Mullerian hormone in women. Hum Reprod Update. 2014;20(3):370-385. doi:10.1093/humupd/dmt062

25. Yue CY, Lu LK, Li M, et al. Threshold value of anti-Mullerian hormone for the diagnosis of polycystic ovary syndrome in Chinese women. PLoS One. 2018;13(8):e203129. doi:10.1371/journal. pone.0203129

26. Hart R, Doherty DA, Norman RJ, et al. Serum antimullerian hormone $(\mathrm{AMH})$ levels are elevated in adolescent girls with polycystic ovaries and the polycystic ovarian syndrome (PCOS). Fertil Steril. 2010;94 (3):1118-1121. doi:10.1016/j.fertnstert.2009.11.002

27. Ranasinha S, Joham AE, Norman RJ, et al. The association between Polycystic Ovary Syndrome (PCOS) and metabolic syndrome: a statistical modelling approach. Clin Endocrinol (Oxf). 2015;83 (6):879-887. doi: $10.1111 /$ cen. 12830

28. Jun TJ, Jelani AM, Omar J, et al. Serum anti-Mullerian hormone in polycystic ovary syndrome and its relationship with insulin resistance, lipid profile and adiponectin. Indian J Endocrinol Metab. 2020;24(2):191-195. doi:10.4103/ijem.IJEM_305_19

29. Li J, Wu Q, Wang CC, et al. Endocrine characteristics, body mass index and metabolic syndrome in women with polycystic ovary syndrome. Reprod Biomed Online. 2019;39(5):868-876. doi:10.1016/j.rbmo.2019.06.014

30. Merino PM, Villarroel C, Jesam C, et al. New diagnostic criteria of polycystic ovarian morphology for adolescents: impact on prevalence and hormonal profile. Horm Res Paediatr. 2017;88(6):401-407. doi:10.1159/000481532

\section{Publish your work in this journal}

Diabetes, Metabolic Syndrome and Obesity: Targets and Therapy is an international, peer-reviewed open-access journal committed to the rapid publication of the latest laboratory and clinical findings in the fields of diabetes, metabolic syndrome and obesity research. Original research, review, case reports, hypothesis formation, expert opinion and commentaries are all considered for publication. The manuscript management system is completely online and includes a very quick and fair peer-review system, which is all easy to use. Visit http://www.dovepress.com/testimonials.php to read real quotes from published authors. 